\section{Leberzellkarzinom und TACE: Korrelieren radiologische Response und Überleben?}

Für die Beurteilung des Ansprechens solider Tumoren gibt es verschiedene Kriterien. Wie gut sie bei nicht resezierbarem hepatozellulärem Karzinom mit dem tatsächlichen Überleben korrelieren, wurde nun untersucht.

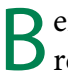
eim hepatozellulären Karzinom korreliert der tatsächliche klinische Nutzen einiger Therapien kaum mit den Ergebnissen konventioneller Untersuchungen. 120 Patienten mit nicht resezierbaren Tumoren wurden mit transarterieller Chemoembolisation mit Doxorubicin freisetzenden Beads (DEBTACE) behandelt. Die mediane Dauer bis zum anschließenden kontrastverstärkten dynamischen MRT betrug 33,5 Tage. Das Tumoransprechen wurde mithilfe der WHO-Kriterien, der RECIST 1.1-, der European Association for the Study of Liver (EASL)- und der modifizierten RECIST (mRECIST)-Kriterien beurteilt.
Bei den Ansprechraten ergaben sich deutliche Unterschiede: Das nach WHO und RECIST 1.1 beurteilte Ansprechen korrelierte schlecht mit dem Überleben. Die Ergebnisse spiegelten nicht einmal wieder, dass Patienten mit vollständigem Ansprechen länger leben als Patienten mit partiellem Ansprechen oder stabiler Erkrankung. Bei den mRECIST- und EASL-Kriterien korrelierten dagegen sowohl das Ansprechen der Zielläsion als auch das Gesamtansprechen signifikant mit dem Überleben ( $\mathrm{p} \leq 0,0001)$. Responder nach den WHO- und RECIST-1.1Kriterien hingegen hatten sogar ein kürzeres Überleben als Nonresponder.
In der Vorhersage des Überlebens war mRECIST besser als die EASL-Kriterien, weil der Unterschied im Überleben zwischen Respondern und Nonrespondern bei den mRECIST-Kriterien statistisch signifikant war $(p=0,013)$, nicht aber bei den EASL-Kriterien ( $\mathrm{p}=0,064)$.

Fazit: Die EASL- und mRECIST-Kriterien für das Ansprechen des Tumors nach der DEB-TACE eignen sich zur Vorhersage des Überlebens, nicht aber die WHO- und die RECIST-1.1-Kriterien. Das Ansprechen nach mRECIST korrelierte stärker mit dem Überleben als das Ansprechen anhand der EASLKriterien.

Judith Neumaier

Prajapati $\mathrm{HJ}$ et al. mRECIST and EASL responses at early time point by contrast-enhanced dynamic MRI predict survival in patients with unresectable hepatocellular carcinoma (HCC) treated by doxorubicin drug-eluting beads transarterial chemoembolization (DEB TACE). Ann Oncol. 2012;24(4):965-73.

\section{HCC: Zwei Tumormarker für Prognose relevant}

Nur 10-15\% der Patienten mit hepatozellulärem Karzinom (HCC) sind Kandidaten für eine kurative Resektion, und selbst hier ist die Prognose mit einem krankheitsfreien Überleben (DFS) nach fünf Jahren von 25-30\% schlecht.

A uf der Suche nach Prognosefaktoren untersuchten Kollegen in Korea retrospektiv die Bedeutung der präoperativ gemessenen Tumormarker Alphafetoprotein (AFP) und Des- $\gamma$-Carboxyprothrombin (DCP) für die Vorhersage eines Rezidivs nach kurativer Resektion eines Hepatitis-B-abhängigen HCC. Dazu unterteilten sie 267 Patienten in zwei Gruppen: Gruppe I: AFP $\geq 20$ ng/ dl, DCP $\geq 40 \mathrm{mAU} / \mathrm{ml}$; Gruppe II: einer oder beide Werte unterhalb dieser

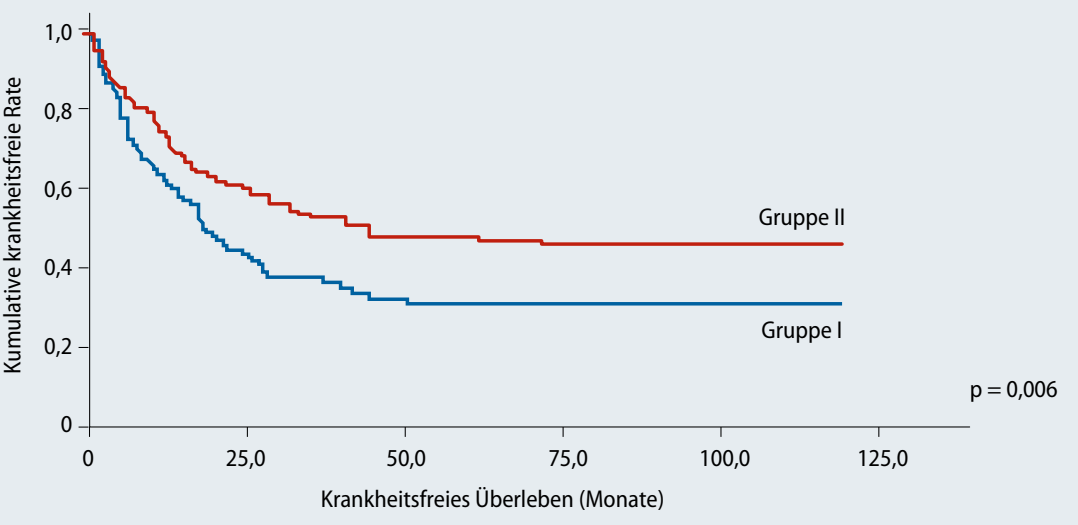

Abb. : Das krankheitsfreie Überleben war in Gruppe I (AFP $\geq 20 \mathrm{ng} / \mathrm{dl}, \mathrm{DCP} \geq 40 \mathrm{mAU} / \mathrm{ml}$ ) deutlich kürzer als in Gruppe II, in der AFPund DCP präoperativ unter diesen Grenzwerten lag.
Grenzwerte. 102 Patienten (38,2\%) fielen in Gruppe I, 165 (61,8\%) in Gruppe II.

Binnen median 69 Monaten entwickelten 154 Patienten (57,7\%) ein Rezidiv, in Gruppe I signifikant mehr als in Gruppe II (66,7 vs. $52,1 \%$; $=0,029)$. In einer multivariaten Analyse waren multiple Tumoren (Hazard Ratio [HR] 2,21, 95\%-Konfidenzintervall [95\%-KI] 1,185$4,121)$ und Tumormarkerwerte entsprechend Gruppe I (HR 1,89; 95 \%-KI 1,080$3,289)$ signifikante Prädiktoren für ein Rezidiv. Das mediane DFS war in Gruppe I mit 20 Monaten signifikant kürzer als in Gruppe II (46,8 Monate; p = 0,006).

Mit der Kombination beider Marker war die Rezidivprognose mit einer Sensitivität von $66,7 \%$ und einer Spezifität von $47,9 \%$ etwas besser.

Fazit: Damit könnte die Kombination dieser beiden Marker, AFP und DCP, als prognostischer Faktor für ein Rezidiv infrage kommen.

Friederike Klein

Chon YE et al. Combined measurement of preoperative a-fetoprotein and des- $\gamma$-carboxy prothrombin predicts recurrence after curative resection in patients with hepatitis-B-related hepatocellular carcinoma. Int J Cancer. 2012; 131(10):2332-41. 